\title{
Dietary Supplements - Essentials for your Heart
}

\author{
Walter Jucker*
}

\begin{abstract}
Today, consumers are actively seeking products that contain health-promoting ingredients, such as nutraceuticals, to improve their health and well-being. The trend of consumers choosing to impact their cardiac health through a dietary approach has been a key growth driver. In this presentation, cardiovascular disease risk factors and a brief overview of the mode of action of dietary supplements that act as essentials for cardiac health are given. With fortified food, different risk factors for cardiovascular disease can be managed. Production strategies for dietary supplements are discussed briefly.
\end{abstract}

Keywords: Cardiovascular disease - Epigallocatechin gallate (EGCG) · Modifiable risk factor · Nutraceuticals · Vitamin E

\section{Introduction}

Cardiac health and cardiovascular risk factors are top health concerns of consumers. The modern way of living is characterized by mental stress situations, a non-balanced diet and sedentariness. Cardiovascular disease dramatically impairs quality of life, causing unfitness, reduction of mobility and shortness of breath. It even can be lifethreatening in the case of myocardial infarction, stroke, angina pectoris and heart failure. Cardiovascular disease constitutes therefore a major social and economic burden.

Among the cardiovascular risk factors are non-modifiable characteristics, such as age, sex, body weight, genetic predisposition and modifiable lifestyle risk factors, e.g. unbalanced diet, smoking, excessive alcohol consumption and lack of physical activity. A third group of risk factors can be modifiable by nutrition.

Most cardiovascular disease risk factors are addressable. You can stop smoking,

\footnotetext{
${ }^{*}$ Correspondence: Dr. W. Jucker
}

DSM Nutritional Products AG, Zweigniederlassung

Werk Sisseln

Head of Compliance

Bau 952

Hauptstrasse 4

$\mathrm{CH}-4334$ Sisseln

Tel.: +4162 8662321

Fax: + 41628662092

E-Mail: walter.jucker@dsm.com maintain a proper body weight, increase aerobic physical activity and keep a balanced diet with reduced dietary sodium and fat intake. In many cases with seriously elevated risk factors, a pharmacological treatment with effective drugs is indicated. To foster the effect of a healthy lifestyle, a full palette of dietary supplements is available on the market. Nutraceuticals and fortified foods are promising answers to the increasing demands for the well-being of an aged population or of a high-risk population, e.g. individuals with a family history of heart disease.

\section{Modifiable Cardiovascular Risk Factors}

Cardiovascular disease (CVD) is a generic term for all pathological changes affecting the heart or blood vessels. Clinical manifestations of CVD are coronary heart disease and stroke. The main underlying process which gives rise to CVD is athero-

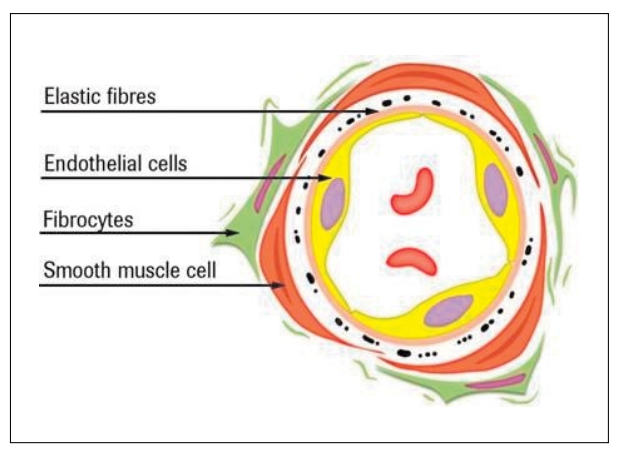

Fig. 1. Schematic diagram of the endothelium sclerosis, which is a pathological process of deposition and hardening of lipids in arteries. Closely connected with atherosclerosis is the function of the endothelium. The endothelium is a single layer of cells lining all blood vessels. It plays a key role in vasoconstriction/vasodilation, coagulation/anticoagulation, inflammation/antiinflammation and oxidation/antioxidation. Fig. 1 shows a schematic illustration of the endothelium.

Examples of modifiable cardiovascular risk factors are hypertension, cholesterols, elevated serum triglycerides, hyperglycemia/diabetes, obesity, hyperhomocysteinemia, elevated thrombogenic factors and the presence of inflammation markers. Some basic information on these risk factors is given below.

\subsection{Hypertension}

Optimal blood pressure is $<120 / 85 \mathrm{~mm}$ $\mathrm{Hg}$ for the systolic and diastolic phases respectively. Hypertension is characterized by a pressure of $>140 / 90 \mathrm{~mm} \mathrm{Hg}$. Hypertension is accompanied by functional changes in several organs and the endothelium. The relative risk of stroke for persons with high blood pressure is ten-fold higher

\subsection{Cholesterols}

Lipoproteins carry fats e.g. cholesterols, in blood plasma. Six different types are distinguished. Low-density lipoprotein (LDL) is the so-called bad cholesterol since it can be oxidized and ultimately stored in plaques (deposits in atherosclerotic blood vessels). High levels of LDL correlate with high risk of CVD. The high-density lipo- 


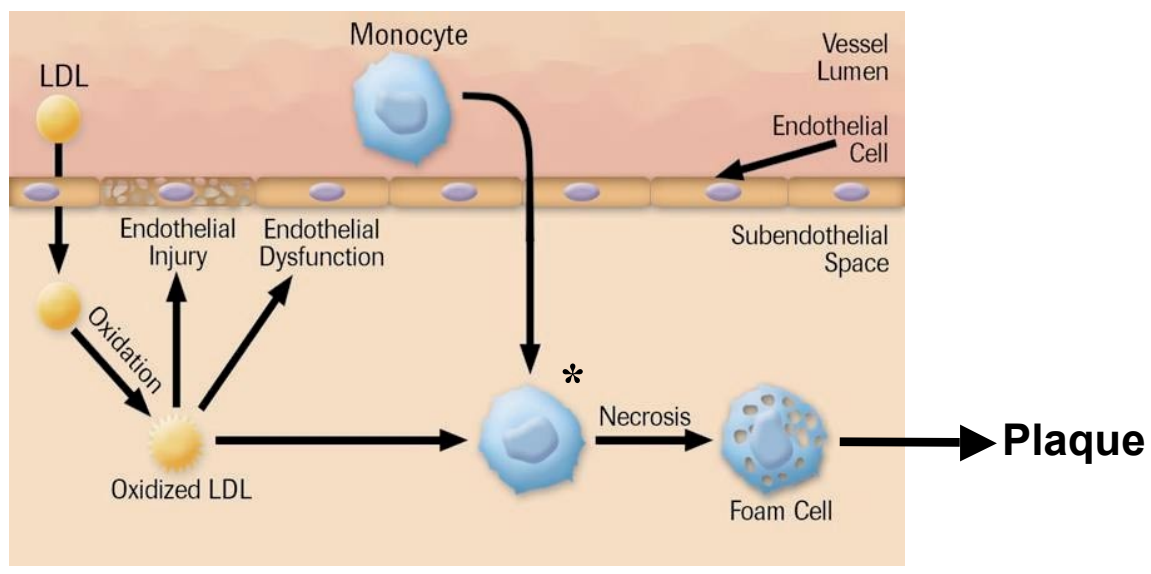

* Monocytes „eat“ oxidized LDL

Fig. 2. Low density lipoprotein (LDL) induced plaque formation in the vicinity of the endothelium

protein (HDL) is the so-called good cholesterol since its concentration is inversely correlated with the risk of CVD. Fig. 2 shows the basic principles.

\subsection{Triglycerides}

Triglycerides are the major form of fat and also the main storage form of fat in the body. More than $95 \%$ of triglycerides arise from food intake. An elevated level of serum triglycerides is associated with increased risk of coronary heart disease. Triglycerides are a component of atherosclerotic plaques.

\subsection{Diabetes}

Diabetes type 1 is characterized by little or no insulin production. Insulin that does not work properly is symptomatic for diabetes type 2 . Both types result in high blood sugar values, so-called hyperglycemia. Diabetes is associated with markedly increased risk of CVD.

\subsection{Obesity}

Obesity is defined as an abnormally high proportion of body fat. Its characteristic trait is a body mass index of $>30$. The primary causes for obesity reside in lifestyle, nutrition, psychological and/or genetic factors. Obesity is a major risk factor for CVD.

If three or more of the above risk factors are present at the same time a metabolic syndrome exists. It results in an increased risk of atherosclerosis.

\subsection{Hyperhomocysteinemia}

Hyperhomocysteinemia is characterized by raised plasma homocysteine levels. It is strongly influenced by genetic factors and dietary nutrients. Homocysteine is metabolized in the human body involving the vitamins folic acid, vitamin $\mathrm{B}_{6}$ and vitamin $\mathrm{B}_{12}$. It is responsible for changes in endothelial function.

\subsection{Thrombogenic Factors}

Thrombogenic factors are involved in blood clotting and include fibrinogen. Fibrinogen is an essential co-factor for platelet aggregation and stimulates migration and proliferation of smooth muscle cells. Increased platelet aggregation and high fibrinogen levels are associated with increased risk of coronary heart disease.

\subsection{Inflammation Markers}

There are several serum inflammatory markers of cardiovascular risk of which $\mathrm{C}$-reactive protein (CRP) is an example. These molecules are released by or expressed on cell surfaces as a response to inflammation. Increasing scientific evidence for a fundamental role of inflamContribute to Metabolic Syndrome mation in mediating all stages of atherosclerosis is currently being discussed.

\section{Essential Ingredients to Control Cardiovascular Risk Factors}

To control many of the modifiable cardiovascular disease risk factors a range of supplements and ingredients are available. Table 1 summarizes the portfolio of essential ingredients offered by DSM Nutritional Products Ltd and indicates their health effects. Fig. 3 shows chemical formulas of the set of dietary supplements that positively influence cardiac health. In principle all these essentials can be provided with adequate diets, but meeting all demands is difficult. Therefore fortified food can be a favorable alternative or supplement.

\subsection{Vitamin C}

Vitamin $\mathrm{C}$ is one of the water-soluble vitamins that lowers blood pressure and improves endothelial function. As an antioxidant it protects LDL against oxidation. Epidemiological studies show reduced risk of cardiovascular disease in individuals with higher intake and plasma levels of antioxidants [1].

Inflammation is the process by which the body responds to injury, infection or irritation. Vitamin $\mathrm{C}$ inhibits the activation of an important transcription factor and histamine release. The latter is also an inflammation signal. Furthermore, vitamin $\mathrm{C}$ stimulates the production of interferon.

Table 1. DSM Nutritional Products portfolio of essential ingredients for cardiac health

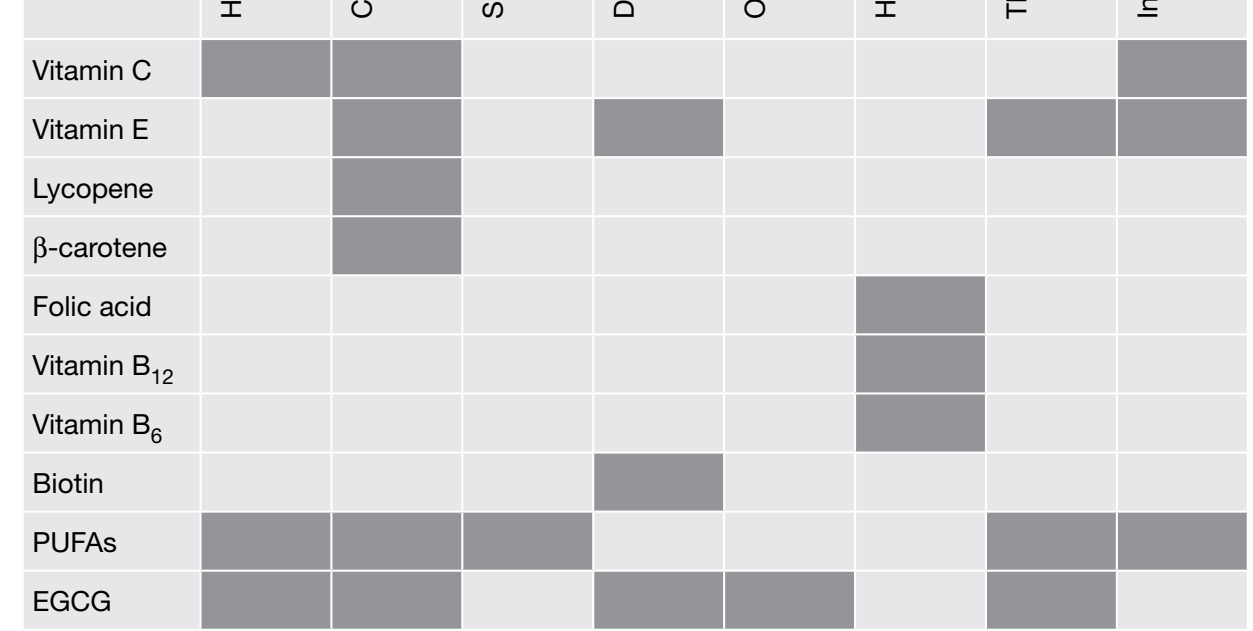




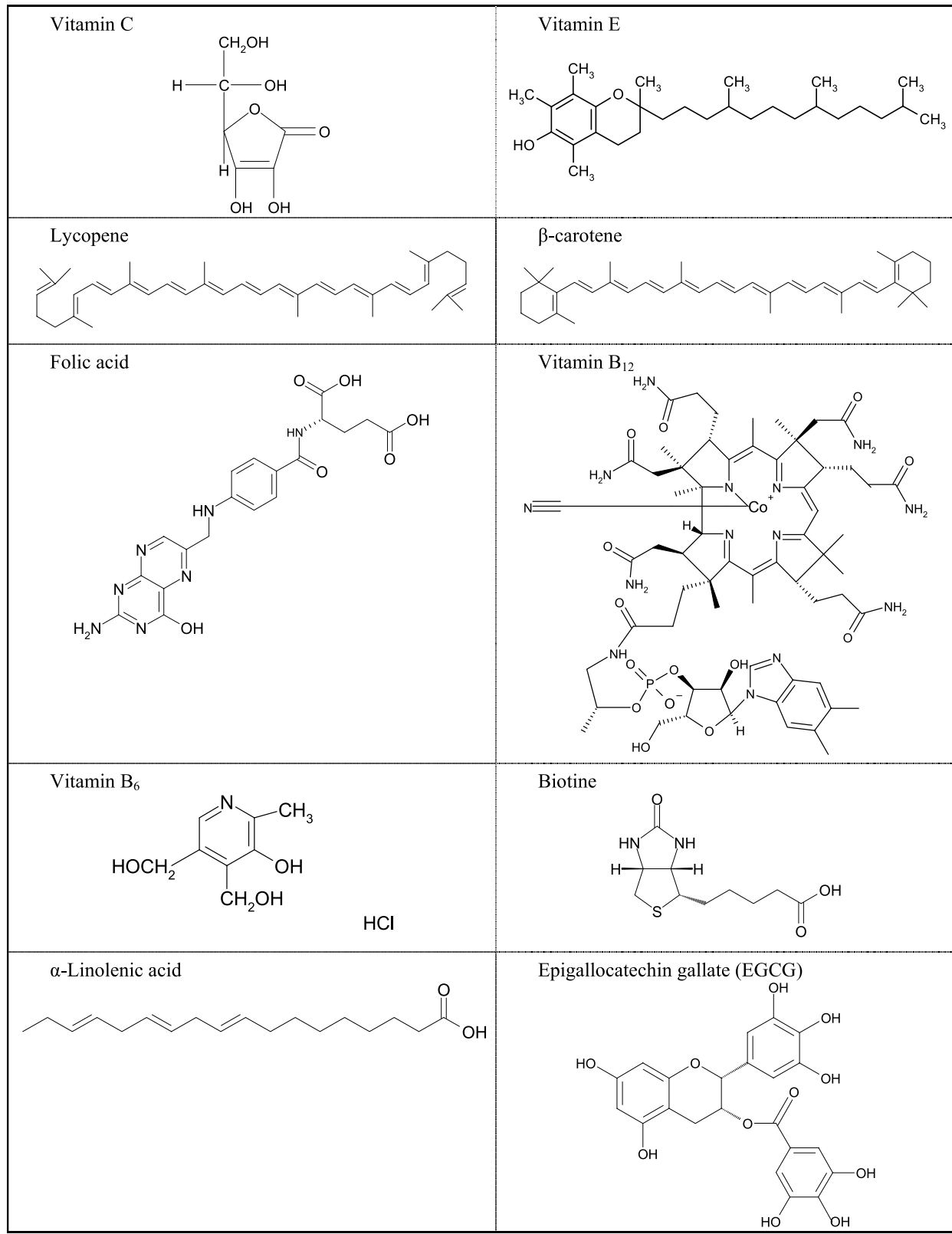

Fig. 3. Chemical formulae of the set of dietary supplements that positively influence cardiac health

\subsection{Vitamin E}

The antioxidative effect of vitamin $\mathrm{E}$ is well known. Thus it acts as a modulator of the distribution between HDL and LDL.

Vitamin E improves insulin sensitivity and consequently reduces the symptoms of Type 2 diabetes. There is evidence that vitamin $\mathrm{E}$ normalizes platelet function and improves endothelial function.

Vitamin E also inhibits platelet aggregation and production of thrombogenic factors such as fibrinogen. Fibrinogen levels rise progressively with the severity of atherosclerosis and after myocardial infarction and stroke [2].

\subsection{Lycopene and $\beta$-Carotene}

Lycopene and $\beta$-carotene are examples of the lipophilic plant pigments named carotenoids. The recommended dietary allowance (RDA) for $\beta$-carotene is $4.8 \mathrm{mg}$ per day [3]. This value is only provided by a diet rich in fruit and vegetables. Due to the long conjugated double-bond sequence, they react as a scavenger of free radicals. This strong antioxidant effect modulates the lipoprotein distribution between HDL and LDL in a way similar to vitamins C and $\mathrm{E}$. In addition to the antioxidative effect, $\beta$-carotene is a complementary source of vitamin A. Lycopene is postulated to lower the risk for prostate cancer through its protective role [4].

\subsection{Folic Acid, Vitamin $B_{6}$ and Vita- $\min B_{12}$}

Epidemiological studies have shown a clear inverse relationship between blood homocysteine levels and intake of folic acid, vitamin $B_{6}$ and vitamin $B_{12}$. Increase of homocysteine is an independent risk factor for cardiovascular disease resulting in higher risk of coronary heart disease and stroke [5]. The beneficial effect of folic acid, vitamin $\mathrm{B}_{6}$ and vitamin $\mathrm{B}_{12}$ is based on their role as cofactors in the metabolism of homocysteine.

\subsection{Biotin}

Biotin, together with vitamin $\mathrm{E}$ and EGCG, influences glucose metabolism [6]. Biotin increases hepatic glucose uptake and decreases simultaneously the rate of gluconeogenesis. It may increase pancreatic insulin secretion. In human studies significantly lowered fasting and after-meal serum glucose levels in diabetics could be demonstrated.

\subsection{PUFAs}

PUFA (polyunsaturated fatty acid) is a collective term for fatty acids with up to 24 carbon atoms linked by single and at least two double bonds with an acid group at the end. Animals and humans lack the enzyme that is responsible for introducing the second double bond in the correct position. Therefore the precursors of all functional $\omega-3$ fatty acids and $\omega-6$ fatty acids, $\alpha$-linoleic acid (18:3 $\omega-3)$ and linoleic acid $(18: 2 \omega-6)$ respectively, are essential. In the nomenclature the first figure indicates the number of carbon atoms, the second figure the number of double bonds and the third figure the position of the first double bond. $\omega-3$ PUFAs are of fish origin and have to be stabilized from oxidation with vitamins $\mathrm{E}$ and $\mathrm{C}$. As a rule of thumb the necessary intake of PUFAs can be reached by eating fish twice per week [7].

$\omega-6$ oils are of plant origin and also have to be stabilized from oxidation with vitamins $\mathrm{E}$ and $\mathrm{C}$. PUFAs modulate many cardiovascular risk factors. Hypertension is positively influenced by improving endothelium response and arterial elasticity as well as by decreasing blood pressure, particularly in mildly hypertensive individuals. PUFAs increase the amount of HDL resulting in a more beneficial HDL/LDL ratio. Serum triglycerides are reduced as shown in $>70$ placebo-controlled human studies. The beneficial effect is consistent and dosedependent. Furthermore they suppress the production of triglycerides in the liver and reduce the synthesis as well as secretion of very low density lipoproteins. A number of studies suggest that PUFAs decrease platelet aggregation and blood viscosity. Several studies show beneficial effects on various inflammatory diseases.

\subsection{EGCG}

EGCG is the abbreviation for epigallocatechin gallate, the most active component of green tea. EGCG is part of the polyphenol fraction, one of the most remarkable components of green tea leaves. The slight astringent and bitter taste of green tea infusions is attributed to these polyphenols. Depending on the species of the tea plant 
and the season for harvesting, EGCG content (dry weight) varies in the region of 10\%. Catechin derivatives are synthesized in tea leaves through malonic acid and shikimic acid metabolic pathways. Gallic acid is derived from an intermediary product produced in the shikimic acid metabolic pathway. Table 2 gives the structure and nomenclature of some components of green tea leaves.

EGCG is an excellent antioxidant by nature; safe and well tolerated with a good bioavailability. Ingested in amounts equivalent to two to four cups of tea, EGCG favors the use of fat as a source of energy leading to a net reduction of fat deposits and, as a consequence, to weight loss [8]. This anti-obesity activity is well documented in animal models and in vitro studies where EGCG modulates various aspects of fat metabolism [9]. For example, it stimulates the $\beta$-oxidation of fatty acids, mainly in the liver, and promotes the expression of an uncoupling protein in adipocytes that leads to extra energy expenditure. Furthermore, EGCG inhibits fat absorption and adipogenesis, the formation of fat-storing cells [10]. EGCG reduces stroke and infarction in rats. It also reduces endothelial damage. Cholesterol levels are positively influenced by lowering total and LDL cholesterol levels in animal studies and by inhibition of LDL oxidation. Several in vivo studies with EGCG extracts indicate lowering of the glucose level and therefore a beneficial effect in diabetics. EGCG inhibits platelet aggregation and reduces thrombogenic factors. This finding correlates with the experience that green tea consumption is inversely associated with risk of stroke. Furthermore, EGCG exhibits an antimicrobial effect. Minimal inhibitory concentrations are in the range between 100 and $500 \mu \mathrm{l} / \mathrm{ml}$.

\section{Manufacturing Strategies}

Principally four different manufacturing strategies are imaginable, namely:

- Extraction and isolation of the active ingredient from natural sources

- Total chemical synthesis

- Chemical synthesis with one or more biological steps

- Microbiological fermentation of the active ingredient

Each of these methods has its inherent advantages and disadvantages. DSM makes use of all these production strategies.

\subsection{Extraction and Isolation of the Active Ingredient from Natural \\ Sources}

EGCG and PUFAs are typical examples for this strategy. EGCG is extracted from green tea leaves. The extraction of polyphenols from tea leaves is performed industri-

Table 2. Structure, occurrence and nomenclature of some components extractable with hot water from green tea leaves

Polyphenol

(+)-Catechin

$(-)$-Epicatechin

1.50

(+)-Gallocatechin

Trace

Occurrence in green tea

Chemical structure $\mathrm{g} / 100 \mathrm{~g}$ dried leaves<smiles>Oc1cc(O)c2c(c1)O[C@H](c1ccc(O)c(O)c1)C(O)C2</smiles><smiles>Oc1cc(O)c2c(c1)OC(c1ccc(O)c(O)c1)C(O)C2</smiles>

(1)

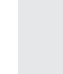

(-)-Epigallocatechin

4.0<smiles>Oc1ccc2c(c1)OC(c1cc(O)c(O)c(O)c1)C(O)C2</smiles>

$\mathrm{OH}$<smiles>Oc1cc(O)c2c(c1)OC(c1cc(O)c(O)c(O)c1)C(O)C2</smiles>

(-)-Epicatechin gallate

$2.8-4.1$<smiles>O=C(Oc1cc(C2Cc3c(O)cc(O)cc3OC2c2ccc(O)c(O)c2)cc(O)c1O)c1cc(O)c(O)c(O)c1</smiles>

(-)-Gallocatechin gallate

0.26<smiles>O=C(OC1Cc2c(O)cc(O)cc2OC1c1cc(O)c(O)c(O)c1)c1cc(O)c(O)c(O)c1</smiles>

(-)-Epigallocatechin gallate<smiles>O=C(Oc1cc(O)c(O)c(O)c1)c1cc(O)c(O)c(O)c1</smiles>

Theanine

1.21<smiles>CCNC(=O)CCC(N)C(=O)O</smiles>

Caffeine

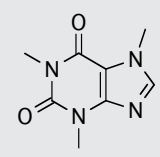


ally by extraction with water and/or lower alcohols. After concentrating the aqueous solution by evaporation, the crude extract is further purified by an additional extraction step with ethyl acetate to isolate a catechin mixture. This mixture is sold as spray-dried green tea extract, which can be of different grades depending on the isolation process. The separation of EGCG from a green tea extract is performed by adsorption chromatography with an inert polyacrylate resin by using polar eluents. The concentrated EGCG fraction is purified by crystallization, centrifugation and drying. The purity of EGCG is increased during the process from about $30 \%$ of commercially available polyphenol concentrates to $>90 \%$. The thermal instability and antioxidative effect of EGCG require carefully selected process conditions. Fig. 4 demonstrates the high purity grade of EGCG. A granulation process is used to obtain the commercial product TEAVIGO ${ }^{\mathrm{TM}}$, which has superior sensory properties. It is completely water soluble, nearly colorless and allows food and beverage fortification with an unchanged taste profile.

PUFAs are extracted from fish oil or plants, deodorized to eliminate the unwanted rancid odor of oxidized by-products and rectified for purification. Under strong anaerobic conditions a beadlet product is produced and sold to the food industry.

The main disadvantage of this production strategy is the enormous consumption of natural raw materials. Plants for extraction of active ingredients are preferably constructed in countries where the raw material is available in large quantities and at the lowest price. Typical production scales for extractions are 10-100 tons per year.

Some consumer groups dislike nature identical products from total chemical synthesis. Therefore niche-products from natural sources are commercially available too. Stereochemically pure D-tocopherol extracted from soy beans or natural $\beta$-car- otene extracted from the fungus Blakeslea trispora are examples.

\subsection{Total Chemical Synthesis}

Chemical synthesis routes for vitamins and carotenoids are based on pioneering research done in the middle of the last century. These classical works are part of chemistry textbooks. Chemical synthesis of vitamins and carotenoids is normally done in world-scale plants. The global market leader is DSM Nutritional Products followed by BASF and Chinese suppliers. The characteristics of these production plants are production quantities in the range between 100 and 20,000 tons a year and mono-product or multiple-product plants but no multi-purpose plants. The typical length of building blocks for the final product is $\mathrm{C} 10$ to $\mathrm{C} 20$. Reactions are often performed in a highly corrosive atmosphere. Very sophisticated reaction types, such as Grignard and Wittig reactions or selective hydrogenations are transferred to industrial reactors. Highly specific catalysts are a must.

The exemplified reaction scheme for vitamin $\mathrm{E}$ is briefly described and formulae are given in the Scheme. The process starts with the batch oxidation of 2,3,6-trimethylphenol (TMP) using pure oxygen to produce 2,3,5-trimethylquinone (TMQ). The safety concept of this production step was published by Kuppinger et al. [11]. TMQ undergoes hydrogenation over a catalyst to produce 2,3,5-trimethylhydroxyquinone (TMHQ). Hydrogenation takes place at elevated temperature and pressure in a loop reactor. A condensation reaction then follows between TMHQ and isophytol over a catalyst. Isophytol itself is produced in an eleven-step chemical reaction synthesis. Tocopherol acetate is produced by acetylation of tocopherol with acetic anhydride. The product is rectified in a distillation column at high vacuum and high temperature prior to being stored in the tank farm. All intermediates are worked up with extraction and distillation operations.
Solvents, acids and catalysts are recovered whenever possible. Waste is incinerated in the on-site boiler house. Other examples for total chemical synthesis are biotin, folic acid and lycopene.

\subsection{Chemical Synthesis with One or More Biological Steps}

A typical representative of this group is vitamin C. It is still produced with the famous Reichstein process, which includes a microbiological production step replacing a number of chemical steps. There is a clear trend in the industry towards direct fermentation of the commercial products.

\subsection{Microbiological Fermentation of the Active Ingredient}

Microbiological fermentation is becoming more and more popular in production of dietary supplements. In contrast to chemical synthesis, fermentations start with non-toxic, cheap starting materials and are run in aqueous media. The very expensive work-up of intermediates after each chemical step is omitted in direct fermentation of the active ingredient. For many years, vitamin $B_{12}$ has been produced with Propionibacter shermanii. According to customer needs, genetically modified organisms for low-cost products are in use, as are strains selected with traditional methods for non-GMO materials. The production of non-GMO products is a new opportunity for niche players.

In recent years enormous efforts have been made to find new syntheses for water-soluble vitamins. Usually, microbiological production processes are breakthrough processes regarding sustainability and costs.

\section{Acknowledgements}

The authors would like to thank Dr. Ingo Koschinski for information on EGCG and Esther Spillmann for excellent administrative support.

Received: September 22, 2006

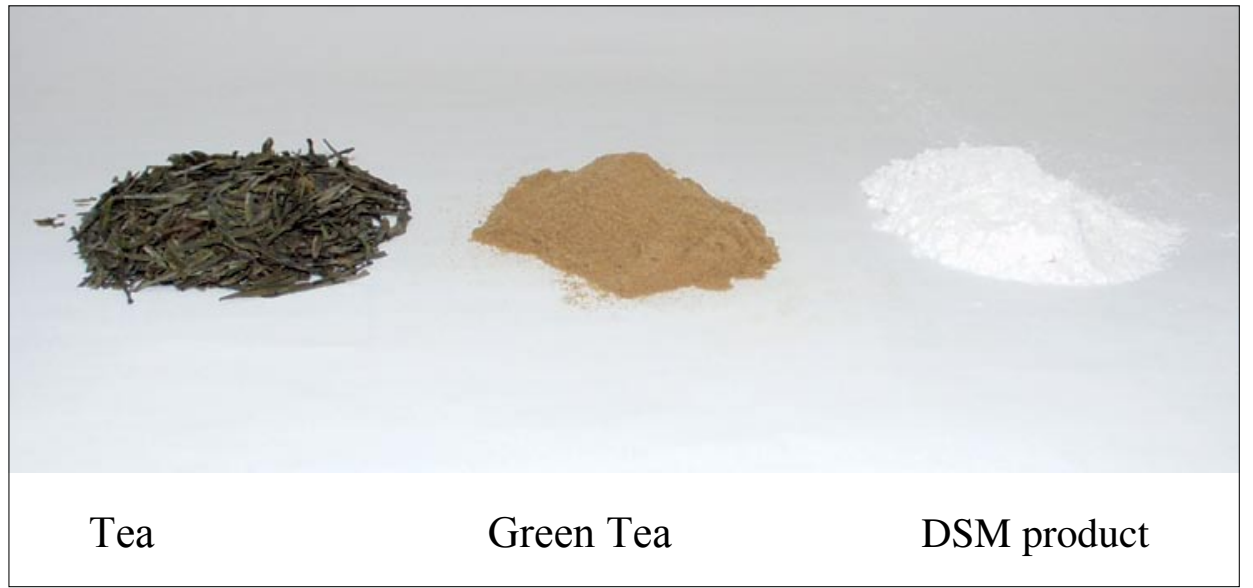




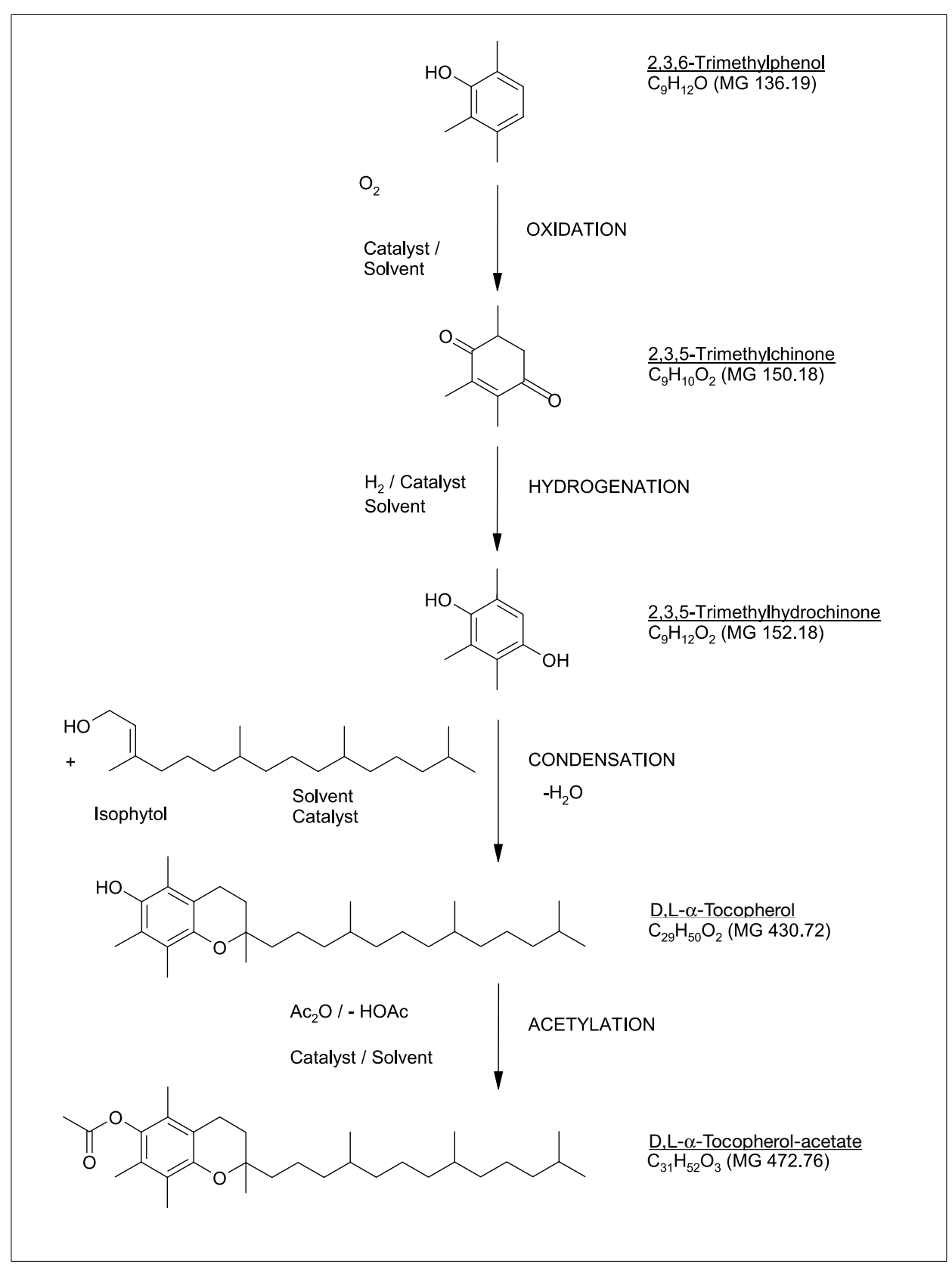

Scheme. Chemical synthesis of vitamin $\mathrm{E}$

[1] I.M. Hajjar, V. George, E.A. Sasse, M.S. Kochar, Am. J. Ter. 2002, 9, 289.

[2] D. Bron, R. Asmis, Int. J. Vitam. Nutr. Res. 2001, 71, 18.

[3] DACH. Referenzwerte für die Nährstoffzufuhr, Umschau Braus GmbH, Frankfurt am Main, 2000, p 69-77.

[4] U.C. Obermüller-Jevic, E. Olano Martin, A.M. Corbache, J.P. Eiserich, A. van der Vliet, G. Valacchi, C.E. Cross, L. Packer, J. Nutr. 2003, 133, 3356.

[5] D.S. Wald, M. Law, J.K. Morris, $B M J$ 2002, 325, 1202.

[6] J.C. Coggeshall, Ann. NY Acad. Sci. 1989, 447, 389.

[7] USDA National Nutrient Database for Standard Reference, www.nal.usda.gov/ fnic/foodcomp/search.

[8] A.G. Dullco, C. Duret, D. Rohrer, L. Girardier, N. Mensi, M. Fathi, P. Chantre, J.
Vandermander, Am. J. Clin. Nutr. 1999 , 70, 1040.

[9] S. Klaus, S. Pültz, C. Thöne-Reineke, S. Wolfram, Int. J. Obes. 2005, 29, 615.

[10] S. Wolfram, D. Raederstorff, Y. Wang, S.R. Teixeira, V. Elste, P. Weber, Ann. Nutr. Metab. 2005, 49, 54.

[11] M. Kuppinger, I. Obermüller, B. Peterhans, Chimia 2005, 59, 693. 